\title{
Capacity Estimation of an Infrastructure Network Carrying HTTP and E-Mail Data based on IEEE 802.11b WLAN Standard
}

\author{
Amandeep Kaur \\ M.Tech Scholar,Dept. ECE \\ SBSSTC, Moga Road \\ Ferozepur, Punjab
}

\author{
Amit Grover \\ Assistant Professor,Dept. ECE \\ SBSSTC, Moga Road \\ Ferozepur, Punjab
}

\begin{abstract}
WLAN IEEE 802.11 Networks should be designed primarily for capacity. In a typical infrastructure WLAN network, now it demands more devices to connect in single network since business-critical applications rely on a Wi-Fi network also the amount of data transmitted wirelessly has significantly increasing which includes voice calls made over Wi-Fi, HD video streaming through $\mathrm{Wi}-\mathrm{Fi}$, file access and storage space in the cloud etc. In our work, we have evaluated the performance of a typical WLAN network working on the principle of IEEE $802.11 \mathrm{~b}$. The scenarios have been figured and analyzed using HTTP \& Email in DLL, PHY \& TPL layers. The statistics calculated are link throughput and application throughput using NetSim simulator.
\end{abstract}

\section{Keywords}

WLAN, IEEE 802.11, IEEE 802.11b, HTTP \& Email, Direct Sequence Spread Spectrum (DSSS), Carrier Sense Multiple Access/ Collide Avoidance (CSMA/CA), forward error correction (FEC).

\section{INTRODUCTION}

Wireless computing is a rapidly emerging technology providing users with network connectivity without being tethered off of a wired network [1], [13]. Wireless Local Area Network have become increasing popularity in networking computers, and replacing need for wiring and extending the service of a LAN backbone [12]. In WLAN mobile users can communicate with each other through technology for networking computers, and replacing need of radio waves. IEEE has developed IEEE 802.11 standard for Wireless Local Area Networks. IEEE 802.1 WLAN has experienced wonderful growth in last ten years. The first internationally WLAN standard IEEE 802.11 introduced in 1997. The IEEE 802.11 standard defines two types of networks; ad hoc and infrastructure. The Basic Service Set is the fundamental building block of the IEEE 802.11 architecture. In BSS a group of stations use the same medium access protocol and the same frequency band [2], [4]. In an ad hoc network stations are grouped to form a single BSS for the purpose of internetworked communications without the help of a base station. Infrastructure networks are recognized to offer some specific services and range extension. Infrastructure networks are recognized using Access Points (APs).

\section{WLAN STANDARDS}

Institute of Electrical and Electronics Engineers (IEEE) develop standard IEEE 802.11 for WLAN. The scope of this standard is to develop a medium access control and physical layer specification for wireless connectivity for fixed, portable, and moving stations within a local area [15]. One of the most commonly deployed wireless technique for WLANs are IEEE802.11 [6] [7] [8]. This standard is used to provide wireless connectivity to equipment, stations that require rapid deployment or automatic machinery, or which may be mounted on moving vehicles, or which may be portable within a local area. For local area communication, this standard provides regulatory bodies by means of standardizing access to one or more frequency bands.

\section{a. IEEE 802.11a}

$802.11 \mathrm{a}$ is based on OFDM waveform at $5.8 \mathrm{GHz}$. It offers protocols that allow transmission and reception of data at rates of 1.5 to $54 \mathrm{Mbit} / \mathrm{s}$.

\section{b. IEEE 802.11b}

IEEE $802.11 \mathrm{~b}$ is the slowest and least expensive existing standard. Due to its low cost, initially $802.11 \mathrm{~b}$ was the most popular standard. But it loses its popularity as faster standards get less expensive. The $802.11 \mathrm{~b}$ standard used DSSS (Direct Sequence Spread Spectrum) modulation technique [11]. IEEE $802.11 \mathrm{~b}$ has maximum data rate $11 \mathrm{Mbps}$. IEEE $802.1 \mathrm{~b}$ uses CSMA/CA medium access technique [9]. Devices using tis standard experience interference from other products operating in the $2.4 \mathrm{GHz}$ band. Microwave ovens, cordless telephones, Bluetooth devices and radio equipments are the devices that operate in $2.4 \mathrm{GHz}$ frequency range [13].

\section{c. IEEE 802.11g}

IEEE802.11 uses OFDM based transmission method that is also used in IEEE 802.11a. It works in the $2.4 \mathrm{GHz}$ band. It operates at a maximum physical layer data rate of $54 \mathrm{Mbit} / \mathrm{s}$ exclusive of FEC codes, or about $22 \mathrm{Mbit} / \mathrm{s}$ average throughputs [3].

\section{SIMULATION SETUP}

NetSim is network simulation software for protocol modeling and simulation, allowing you to analyse computer networks with supreme depth, power and flexibility. NetSim is being used by the world's most prestigious institutions for network lab experimentation and research. NetSim has an open, modular and flexible architecture NetSim which enables user to call into NetSim user generated libraries to run network scenarios.

In our scenario, we have created a WLAN enterprise network based on IEEE 802.11 b standard using NetSim Simulator \& connected three access points with a single Ethernet switch. The access points are further connected to various wireless client nodes. We have taken nine wireless client nodes. Two types of links are used to connect the devices. Access points are connected to switch through wired PPP (point to point) full duplex mode link, here we also considered bit error rate factor due to some losses present in the real system which 
affects the Quality of Service whereas wireless link are connected with point to multipoint half duplex system with 11 Mbps uplink \& downlink speeds and we also considered no path loss factor in one subsystem and fading loss in another subsystem. We have applied two applications i.e. HTTP and EMAIL for analysing the system performance by the means of link throughputs \& application throughputs.

\section{RESULT AND DISCUSSION}

At the end of a simulation run network performance related metrics are collected. Simulation experiment include metrics like throughput, collision counts, simulation time, packets transmitted, packets dropped, packets errored, etc, and their respective values_at the end of the completion.

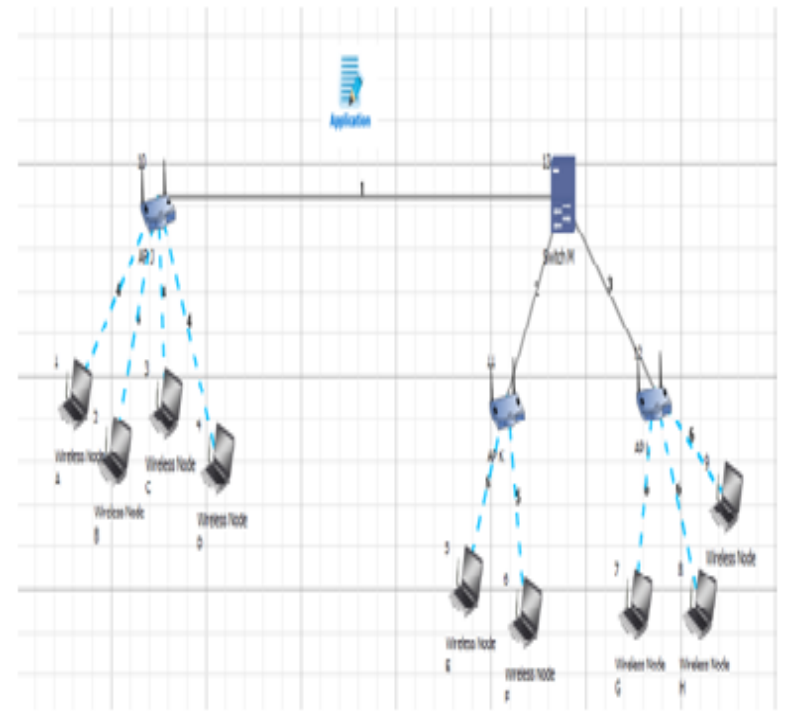

Fig. 1: Network Scenario

\section{a. Wired Link type:}

PPP (Point to Point): Wires link has been established between the Access point \& switch. In Networking, to establish a direct link between two networking devices PPP protocol is used.

\section{b. Wireless Link Properties:}

AP2 \& AP3 are connected through wireless link with nodes in half duplex mode, without any path loss. Nodes 1,2,3,4 are connected with AP1 through wireless link in half duplex mode with fading loss only.

\section{c. Congestion Control Algorithm}

In this scheme we've used TEHOE congestion control algorithm, in this the retransmission is fast. The algorithm works based on duplicate acknowledgement i.e. when it receives three acknowledgements which is the indication of segment loss, that segment is retransmitted immediately without waiting for timeout. Window size represents the number of segments that can be transmitted at a time by the sender [5], [10]. We have used IPv4 addressing in our scheme. The Internet Control Message Protocol offers an echo mechanism by which a host may solicit a response from a gateway by sending message at a reasonable rate. Since the messages must be sent fairly often to detect a fault in reasonable time, it can imply an unbearable overhead. The range of ICMP continuous polling time should be between 0 600 .

Path loss:-Here we considered the fading loss into our wireless channel which affects the QoS, due to fading signal can take several different paths among sender and receiver due to reflection, spreading and diffraction and hence called fading.

\begin{tabular}{|c|c|}
\hline Metric & Value \\
\hline Simulation Time(ms) & 500000,00 \\
\hline Packets Transmitted & 198495 \\
\hline Packets Errored & 361 \\
\hline Packets collided & 6229 \\
\hline Bytes Transmitted(Bytes) & 1000506094,00 \\
\hline Payload Transmitted(Bytes) & 84046150,00 \\
\hline Overhead Transmitted(Bytes) & 16004544,00 \\
\hline
\end{tabular}

Fig. 2: Network Metrics

Fig. 2. shows amount of time for which the simulation is run, in our scenario we have taken five hundred seconds for the simulation of our network. 1, 98,495 packets transmitted in the entire network. We have 361 packets are errored which are not delivers successfully and are lost due to collisions between the packets. 6,229 packets collided, which are collided between the transmission processes. Total bytes transmitted including payload and overhead data in our scenario are $100050 \mathrm{~K}$ in the whole simulation process. The total payload count is $84046 \mathrm{~K}$. The total overhead transmitted is $16004 \mathrm{~K}$ during the entire process of communication.

In our project, we have plotted the throughput graphs for both the applications i.e. HTTP \& E-mail. We analyse that the number of bytes transmitted are quite large in this experiment; there are large number of collisions so performance of a $\mathrm{p}$ persistent CSMA/CD network is not optimal with large $\mathrm{p}$ [14]. 


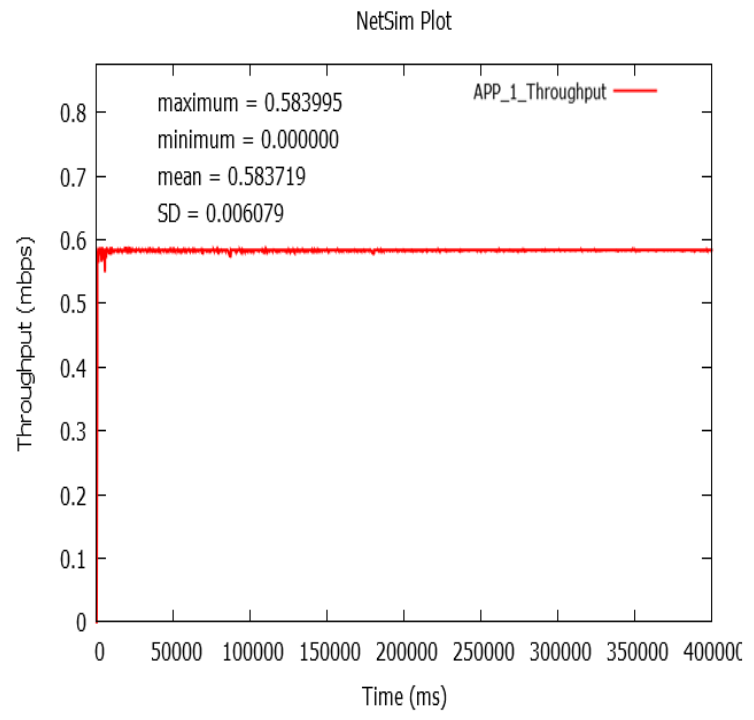

Fig. 3 Application HTTP Throughput

Therefore, In Fig. 3 we have throughput in the range of 0.58 Mbps (Max.) to 0.00 (Min) with a mean value of $0.58 \mathrm{mbps}$ during the whole communications, but as persistence is decreased, the likelihood of collisions decrease and for this reason throughput starts to increase again beyond a certain limit, but then decreases and then stable after 10-20 seconds of simulation. In this case there are not many transmissions because $1 / 11$ the probability of transmitting packets becomes very low and hence, we also noticed that the standard deviation of throughput is at a persistence value of 0.0054 during the whole process.

Similarly, in second application i.e. E-mail transmission the throughput of is gradually increases but initially the throughput high and as the number of collision increases the throughput drop by to some extent for a transitory short time and then stabilise as shown in Fig.4.

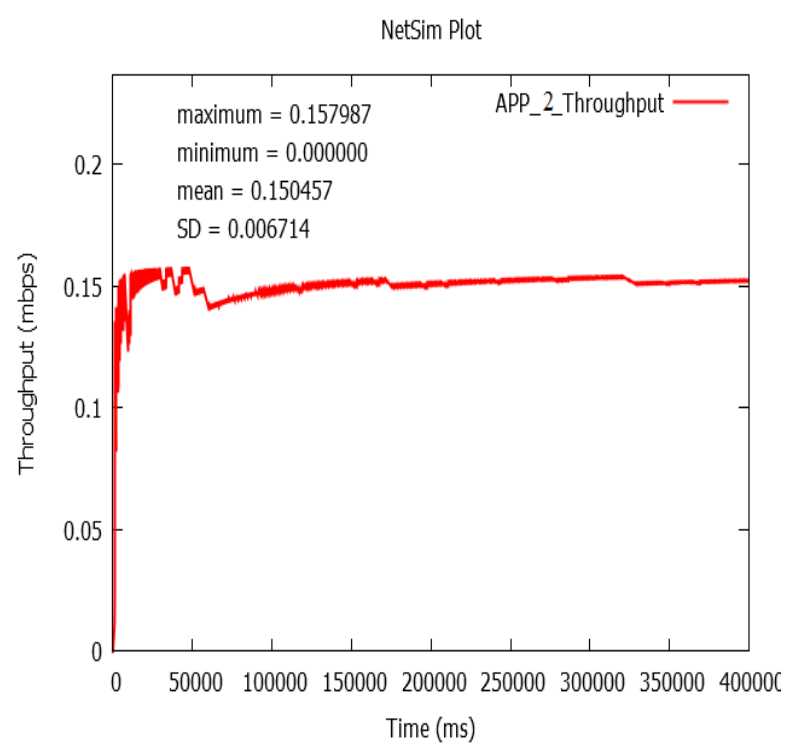

Fig. 4 Application E-mail Throughput

In our network, we also calculate the link throughputs which we have used in the system, Link 1 is the wired node between Switch and access point 1 . Access point switches traffic between wireless client nodes based on 802.11 addresses.
Payload is terminated at AP and transported inside Ethernet frame to switch. Ethernet frame with payload terminated are transmitted by the switch at access points and payload is then transported inside 802.11 frames to end node. In link throughput, we can easily see that the throughput of the link first increases as the load on the link is less and then reduce a bit due to receiving's of the acknowledgements and ARP requests and then stabilizes afterwards. Here we have maximum $1.4 \mathrm{mbps}$ is received because we have considered the high BER in the wired point to point mode of wired link as shown in Fig.5.

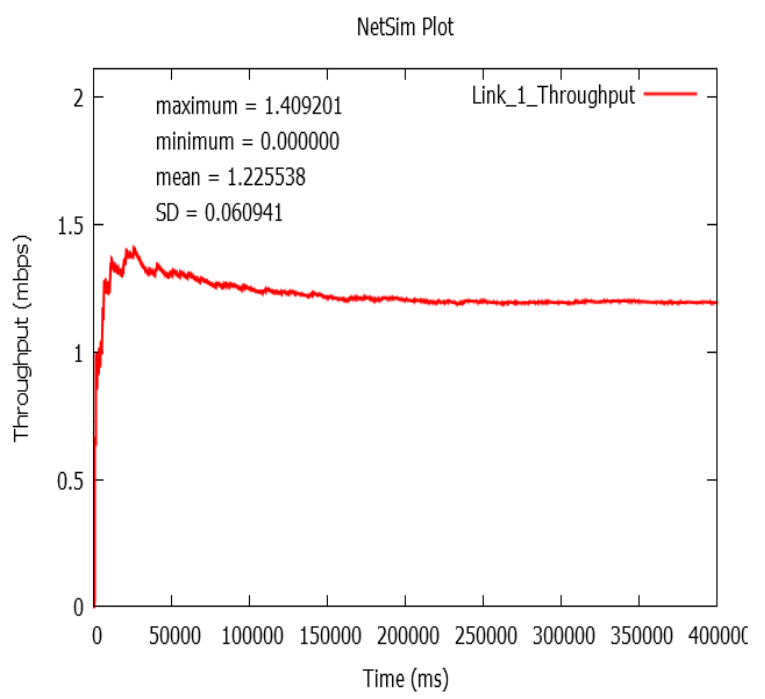

Fig. 5 Link 1 Throughput

Link 2 is wired node between switch and access point 2.As shown in Fig.6 throughput first increase as load is less then stabilize.

NetSim Plot

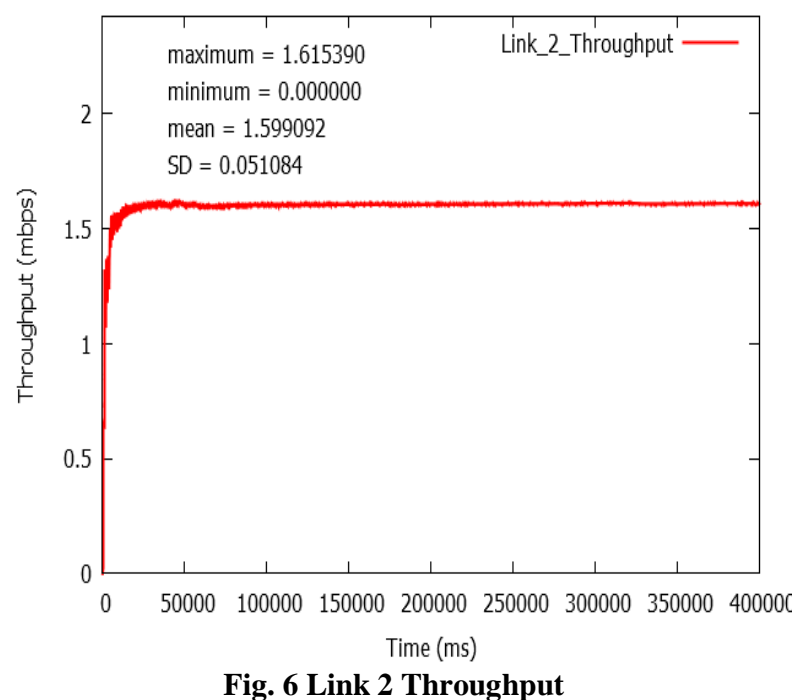

In wireless link 4, as we have considered the fading loss into it, so we get less throughput as compare to the throughput of wireless link 5. Hence, fading affect the quality of service and provides throughput of maximum 2.0 mbps (appox.) transmission speed. The throughput is also depends on the distance between the access point and wireless nodes, since we have created a enterprise network in large area the distance between the AP's and wireless client nodes is also more which significantly reduces the QoS and overall throughput 


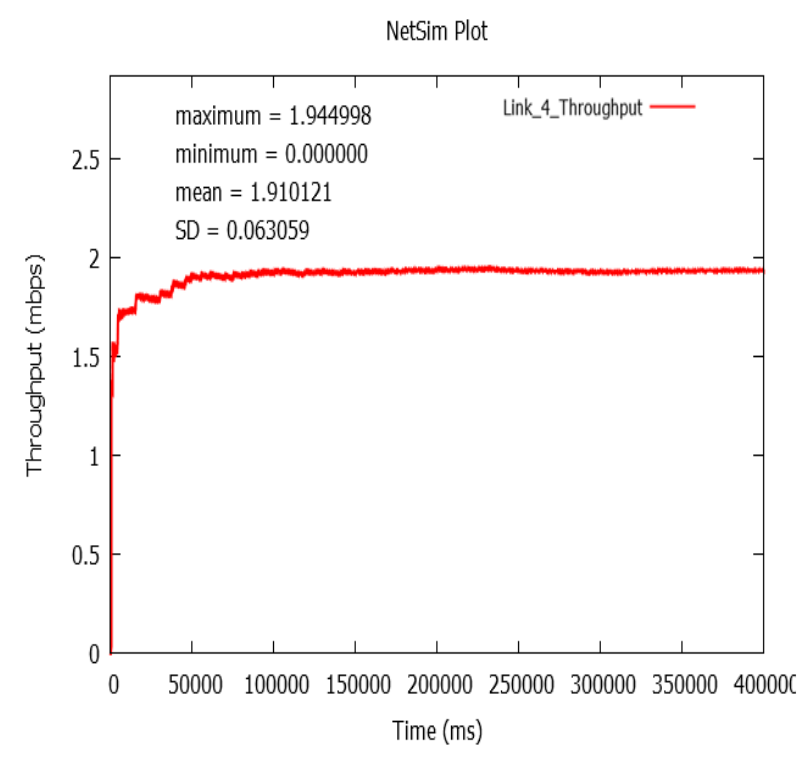

Fig. 7 Link 4 Throughput

In wireless kink 5, since we have not considered the path loss factor into this wireless link between access point \& wireless nodes the throughput we received is much higher than what we have obtained in wireless link 4. Here the throughput first increases gradually and after some variations it becomes stable as shown in Fig 7. Here also the distance between the APs and the wireless nodes affect the QoS and throughput. We have got approx. 4.0.

NetSim Plot

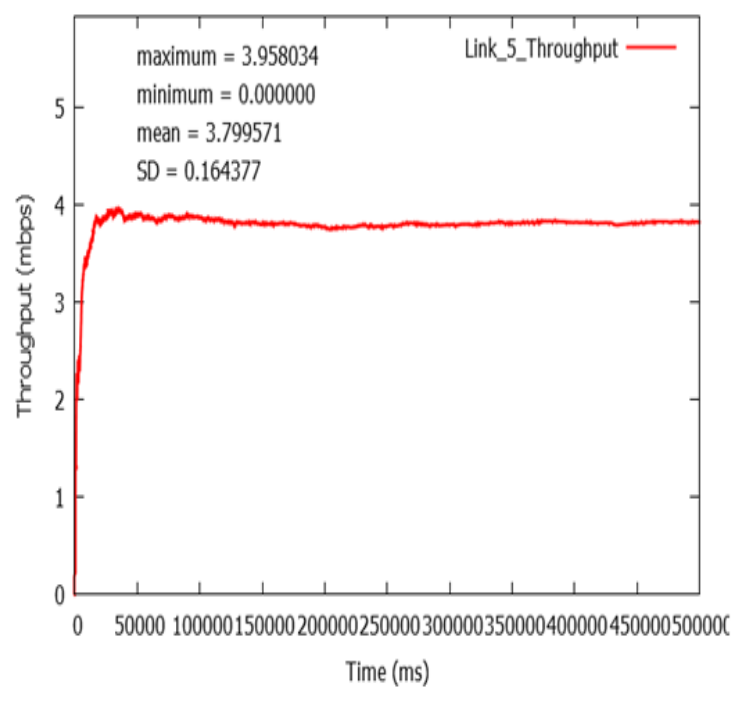

Fig. 8 Link 5 Throughput

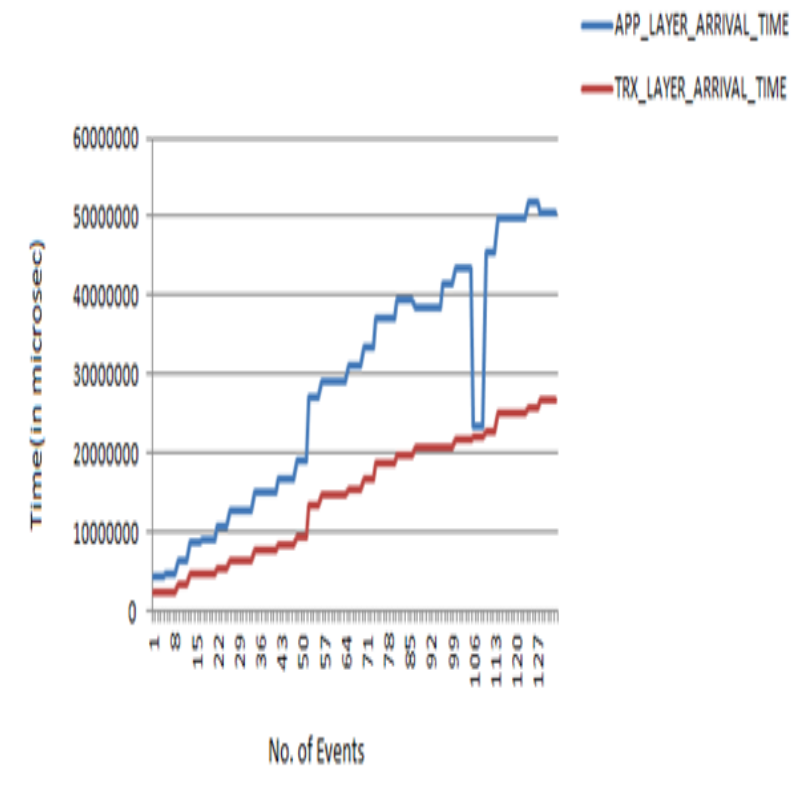

Fig.9 Inter-arrival time of application layer and transport layer

In Fig.9, the inter-arrival time of application layer and transport layers are shown, as the inter arrival time is the time delay between the successive packet, smaller inter arrival time leads more no. of data packets being generated per unit time and vice-versa. The inter arrival time for transport layer increases with increase in number of events. The inter arrival time for application layer

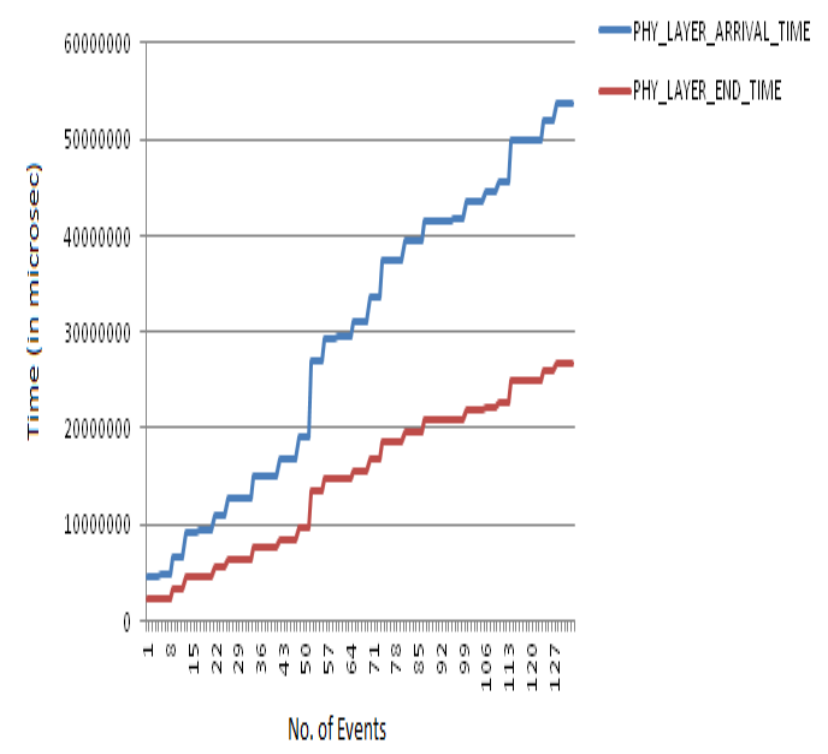

Fig.10 Physical layer arrival time $\&$ end time

In Fig.10 Physical layer arrival time \& end time are shown as per the occurrence of event, the arrival time is also depend upon the distribution. The distribution can either be constant or exponential. The inter-arrival time of a poison process is distributed exponentially and poison process use to model random events, it can be modifies by the user. 


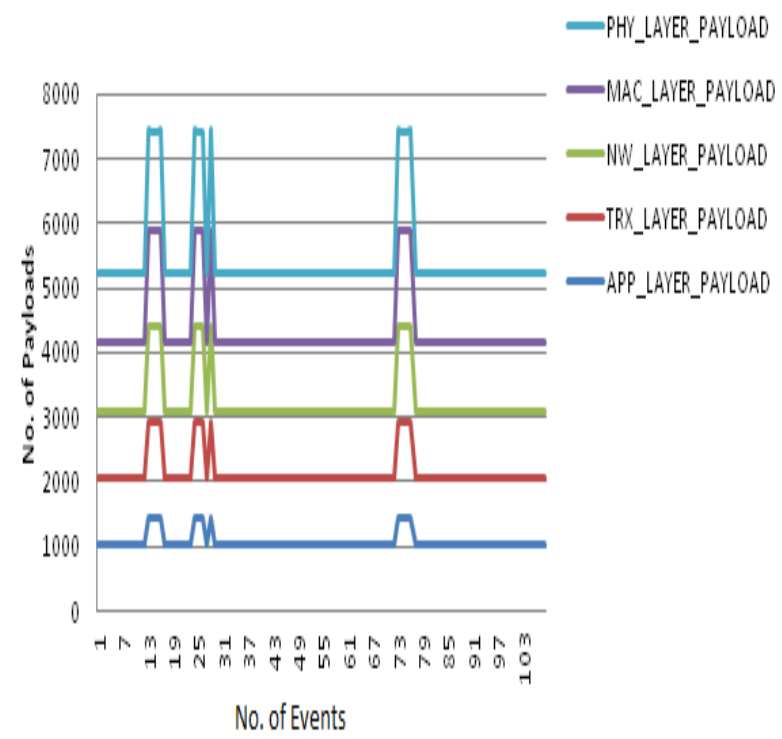

\section{Fig11. Number of payload transmitted from physical to application layer}

In Fig.11, numbers of payload transmitted from physical layer to application layers are shown as per the occurrence of the events in the network, since, payload Transmitted is the total payload transmitted in the entire network in bytes. It is equal to sum of the 'Payload Transmitted' in all the links.

\section{CONCLUSION}

Our simulation results observed that, in WLAN IEEE 802.11 $\mathrm{b}$ based enterprise network, there are various parameters like fading path loss, distance between the access points and wireless clients, types of the wireless \& wired medium, congestion control algorithm in the transmission layer, switching mode etc. which affects the quality of service and other system throughputs. Further it is also seen that the throughput is also depend upon the type of application used. In future we can enhance the quality of services by diminishing the errors by implementing the new congestion control algorithms \& some other factors in the networks which affects the quality of service of a network. We can also implement the same \& other scenarios by using IEEE 802.11 $\mathrm{n}$ latest wireless standards and can implement and analyse some other applications like voice, data, video, email etc in our network.

\section{REFERENCES}

[1] Yibing DENG, Wei HU et al. "Research and Simulation on Application of the Mobile IP

[2] Network" Elsevier Physics Procedia 33 ( 2012 ) 1588 1595

[3] Hiertz, Guido R., et al. "The IEEE 802.11 universe." Communications Magazine, IEEE 48.1 (2010): 62-70.
[4] Ramsey, Benjamin W., et al. "Subjective audio quality over a secure IEEE $802.11 \mathrm{n}$ network." International Journal of Security and Networks 6.1 (2011): 53-63.

[5] Barcelo, Jaume, et al. "Learning-BEB: Avoiding collisions in WLAN." Other IFIP Publications 1 (2011).

[6] Doufexi, Angela, et al. "A comparison of the HIPERLAN/2 and IEEE 802.11 a wireless LAN standards." Communications Magazine, IEEE 40.5 (2002): 172-180

[7] $\mathrm{Na}$, Chen, Jeremy K. Chen, and Theodore S. Rappaport. "Measured traffic statistics and throughput of IEEE 802.11 b public WLAN hotspots with three different applications." Wireless Communications, IEEE Transactions on 5.11 (2006): 3296-3305.

[8] Ott, Jörg, and Dirk Kutscher. "Drive-thru Internet: IEEE 802.11 b for" automobile" users." INFOCOM 2004. Twenty-third AnnualJoint Conference of the IEEE Computer and Communications Societies. Vol. 1. IEEE, 2004.

[9] Jun, Jangeun, Pushkin Peddabachagari, and Mihail Sichitiu. "Theoretical maximum throughput of IEEE 802.11 and its applications." Network Computing and Applications, 2003. NCA 2003. Second IEEE International Symposium on. IEEE, 2003.

[10] Brik, Vladimir, Arunesh Mishra, and Suman Banerjee. "Eliminating handoff latencies in 802.11 WLANs using multiple radios: Applications, experience, and evaluation." Proceedings of the 5th ACM SIGCOMM conference on Internet Measurement. USENIX Association, 2005.

[11] Heusse, Martin, et al. "Performance anomaly of 802.11 b." INFOCOM 2003. Twenty-Second Annual Joint Conferences of the IEEE Computer and Communications. IEEE Societies. Vol. 2. IEEE, 2003.

[12] Vishal Sharma, et al. "Quality of Service (QoS) evaluation of IEEE 802.11 WLAN using different PHYLayer Standards" Elsevier OPTIK, (2012).

[13] Klepser, B-U., et al. "5-GHz and 2.4-GHz dual-band RFtransceiver for WLAN $802.11 \mathrm{a} / \mathrm{b} / \mathrm{g}$ applications." Radio Frequency Integrated Circuits (RFIC) Symposium, 2003 IEEE. IEEE, 2003.

[14] Bhandare, Suryakant, et al. "Performance evaluation of collision avoidance schemes in ad hoc networks." Security and Communication Networks (2014).

[15] Shoab A. Khan. "802.11 Disassociation DoS Attack and Its Solutions: A Survey", 2006 Proceedings of the First Mobile Computing and Wireless Communication International Conference, 09/2006. 